\title{
Bilateral Lumbar Hernias - A Case Report and An Overview of the Current Literature
}

\author{
Mina Cheng* and Canon King-On Chan \\ Department of Surgery, Queen Elizabeth Hospital, China
}

Received: October 11,2017; Published: October 17, 2017

*Corresponding author: Dr. Mina Cheng, Department of Surgery, Queen Elizabeth Hospital, 33 Gascoigne Road, Kowloon, Hong Kong SAR, China, Tel: 852-90933527; Email: minacheng0505@gmail.com

Keywords: Lumbar hernia; Bilateral; Hernia repair

\section{Introduction}

Primary lumbar hernia is a rare clinical entity. So far, around 300 cases have been described in the literature and almost all of them describe repair for unilateral hernias. We present a case of bilateral primary lumbar hernia repair at our institution.

\section{Case Report}

A 79 year old lady, who had enjoyed unremarkable health and was fully independent on her activity of daily living, was presented to us with self-detected bilateral loin swellings for one year. The swellings did not produce any symptoms of pain or discomfort and she denied any history of trauma to the region or symptoms of cough, constipation or urinary outflow obstruction. Physical examination revealed her Body Mass Index (BMI) to be $19.1 \mathrm{~kg} /$ $\mathrm{m} 2$. Abdominal examination found a non-distended abdomen and there was absence of surgical scars or ventral hernias. Bilateral lumbar protrusions were detected on upright (Figure 1), but they were completely reduced in prone position, which was compatible with lumbar hernias. Computer tomography (CT) scan demonstrated bilateral lumbar hernias just below the twelfth ribs. Retroperitoneal fat was seen inside the right hernia sac and a segment of descending colon was seen inside the left hernia sac (Figure 2). She later consented to an elective repair of her bilateral lumbar hernias under elective settings.

Figure 1.

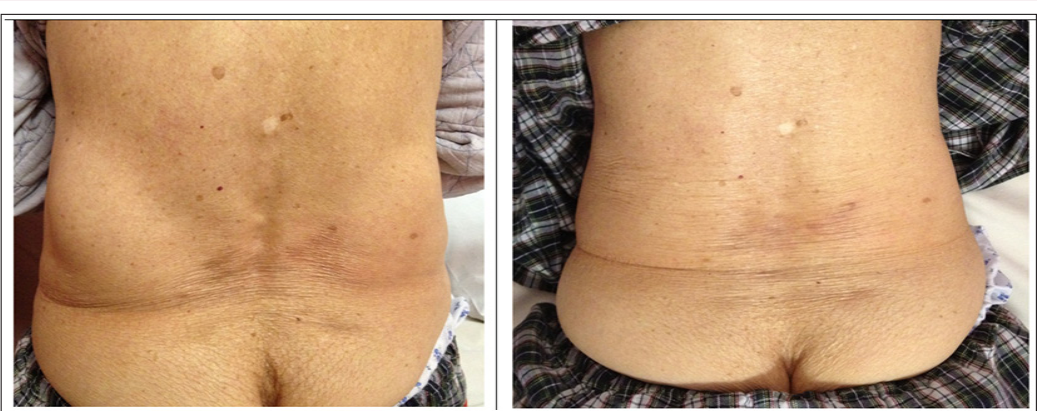

Figure 2.
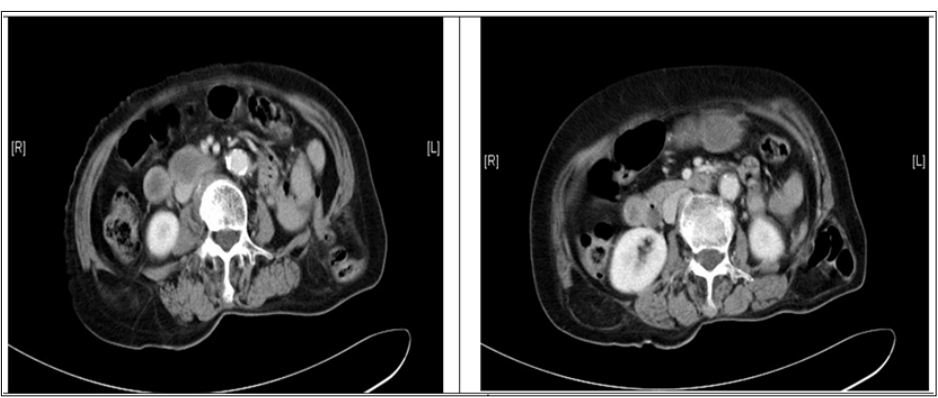


\section{Perioperative Management}

She was admitted one day before the operation for assessment by the list anesthetist as well as for bowel preparation. Klean-Prep polyethylene glycol solution (Helsinn Healthcare S.A., Switzerland) was given in case bowel resection was indicated. She started fasting eight hours before the operation. Amoxicillin and clavulanic acid were given by intravenous route during induction of anesthasia. Repair of the bilateral lumbar hernias was performed under general anesthesia with endotracheal intubation. The patient was first positioned in right decubitus for the repair of the larger left side lumbar hernia. Although prone position had obvious advantage of allowing both sides repair to be performed without position change, we decided to carry out the lumbar hernias' repair one side at a time in their respective decubitus positions in order to avoid the unnecessary risks associated with prone anesthesia [1,2].

\section{Operative Findings}

Both lumbar hernias were from the superior lumbar triangle. The fascial defects measured $3 \times 3 \mathrm{~cm}$ in size over the left side, and $1 \mathrm{x} 1 \mathrm{~cm}$ over the right side. A segment of descending colon was found in the hernia sac over the left side, and perinephric fat was noted in that over the right side.

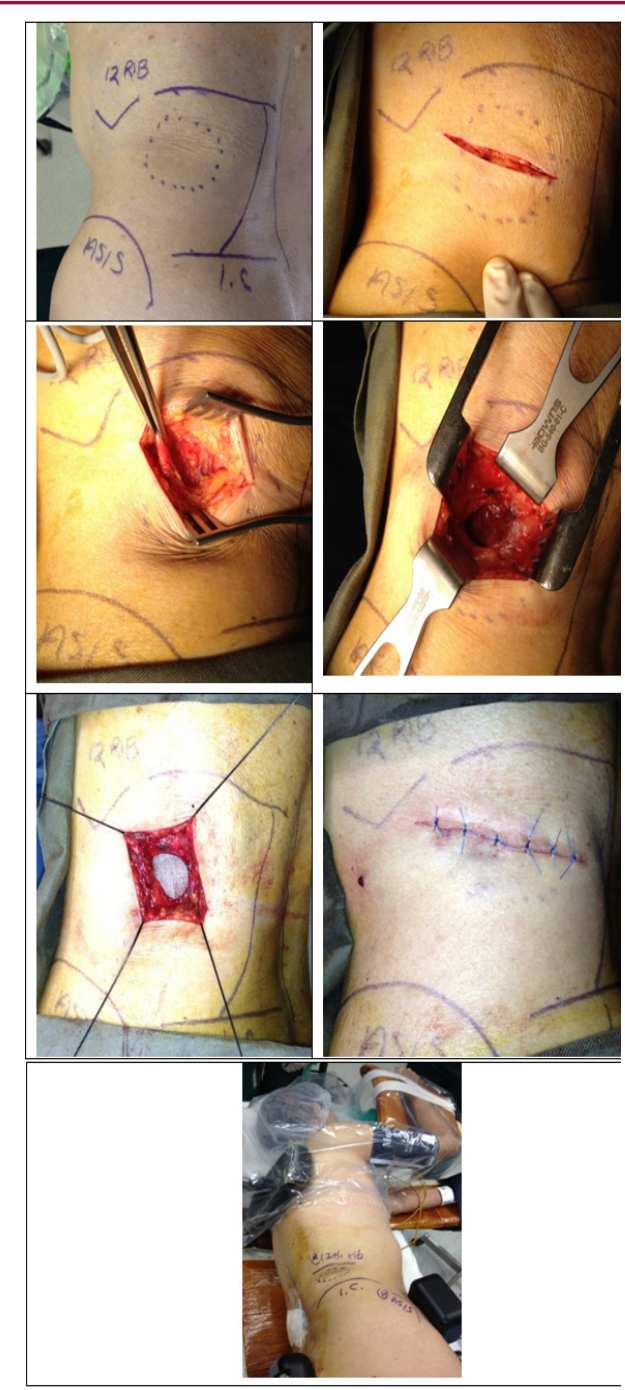

Figure 3.

\section{Method Of Repair}

Due to the rarity of bilateral primary lumbar hernias, as well as the limited experience available in the current literature on laparoscopic approaches in this clinical entity, we decided for open repair as it remained to be simple, safe and effective [3,4]. An oblique incision was made over the left lumbar hernia. The Latissimus dorsi muscle was retracted cranially and the hernia content was reduced. A $7 x 7 \mathrm{~cm}$ Prolene polypropylene mesh (Ethicon, USA) was placed in 'sublay' manner beneath the lumbar fascia defect. The mesh was fixed by non-absorbable monofilament stitches applied superiorly to the periosteum of the twelfth rib, anteriorly to the internal oblique muscle, and posteriorly to the quadratus lumborum (Figure 3). The wound was then closed in layers after hemostasis had been attained. Following the completion of the left lumbar hernia repair, the patient was turned to left decubitus position and the same procedure was repeated on the right lumbar hernia. The operative blood loss was $30 \mathrm{ml}$ and the operation took 138 minutes. Postoperatively the patient recovered uneventfully and diet was resumed immediately after the patient recovered from the effect of anesthesia on the same day. The patient was prescribed ondemand oral paracetamol 500mg every 6 hours for the first three days after the operation. She was fully mobilized on the next day after the operation and was discharged on post op day 2 . There was no recurrence detected on follow-up at 6 months.

\section{Discussion}

Queen Elizabeth Hospital is a tertiary referral surgical center with a catchment population of one million. Our institution carry out approximately 1200 cases of hernia repair yearly, but the incidence of primary lumbar hernia is extremely low accounting for just one case per year $(0.09 \%)$. Lumbar hernia is a rare condition. It was estimated that only around 300 cases have been reported in the literature $[5,6]$. However, the incidence of bilateral lumbar hernias was mostly described in the pediatric population, in which case they were classified as congenital and usually associated with other inborn abnormalities [7-11]. Only a few reports have described experience on the acquired-type bilateral primary lumbar hernias, which are found in adults [3,9]. Despite its rarity, acquired lumbar hernias possess specific clinical features. Acquired lumbar hernias account for around $80 \%$ of cases [12]. They are classified as primary (spontaneous) or secondary, depending on the existence of a causal factor (previous incision, trauma or infection). Approximately $55 \%$ of acquired lumbar hernias belong to the primary type [13]. Primary lumbar hernias are more often found on the left side and in the upper lumbar triangle [14]. They are precipitated by conditions associated with increased intra-abdominal pressure, such as strenuous physical activity or chronic bronchitis.

The commonest contents of a lumbar hernia include retroperitoneal fat, small and large bowel, omentum, caecum, appendix, stomach, ovary, spleen and kidney $[15,16]$. Nonetheless, these reports include cases of post traumatic or incisional lumbar hernias where the hernial sac may be of substantial size. The diagnosis is obtained by clinical examination, and can be supplemented by CT imaging, where the sensitivity is approximately 98\%. Some have also found MRI useful in confirming lumbar hernia 
defects $[15,17,18]$. In contrary to the aforementioned, albeit our patient belongs to the acquired primary type of lumbar hernia, she did not have the typical risk factors for lumbar hernias, hence the development of her lumbar hernias was likely a result of anatomical alterations of the posterior abdominal wall caused by aging [6].

Most lumbar hernias increase in size with time, and overall, there is a $25 \%$ risk of incarceration and $8 \%$ rate of strangulation. It is therefore generally agreed that lumbar hernias should be repaired early [19]. Primary repair is challenging due to inadequate fascia around the defect making tensionless repair difficult [20]. Various primary repair [21], tissue flaps [9,17,22,23] and mesh repairs (including laparoscopic trans abdominal [3,24-27] and retroperitoneoscopic approaches [19,28-30] had been described. In view of the high failure rates observed in primary repair with facial closure, the principle of tensionless repair by the use of prosthesis became more popular in Lumbar hernia repairs. This has led to more recent publications on the use of pros $\neg$ thetic mesh to bridge the defect and minimize tension, which was associated with a lower recurrence rate [31,32]. As tension-free methods became more popular, reports on the use of different prosthesis design and modification followed.

Losanoff and Kjossev reported on the use of a cylindrical shape polypropylene mesh plug. It was proven to promote powerful fibroblastic response to repair the wall defect [17]. On the other hand, Armstrong and co-workers reported the use of the Ventralex patch, which combines a layer of PTFE with a smaller polypropylene mesh. This mesh allowed the surgeon to perform with a small incision and to place the mesh within the peritoneum with polypropylene straps abutting the patch to the fascia, and was associated with a quick recovery as well as good long-term outcome [18]. Solaini and co-workers reported the use of a dart mesh (Bard Mesh Dart, small monofilament knitted polypropylene), which was sutured medially to the quadratus lumborum, externally to the internal oblique, and superiorly to the periosteum of the twelfth rib and the serratus posterior-inferior. Like other similar studies, the author observed a quick recovery and no recurrence upon followup at 11 months [33]. These reports have revolutionized the use of a mesh in achieving tensionless repair in lumbar hernias. It was based on this and our centre experience that open repair using prosthesis was selected for our patient as it is simple, safe and effective. We anchored a Prolene polypropylene mesh (Ethicon, USA) in sublay manner with Nylon stitches superiorly to the periosteum of the twelfth rib, anteriorly to the internal oblique muscle and posteriorly to the quadratus lumborum. The sublay or underlay placement of a mesh is scientifically sound as buttressing the mesh posteriorly takes full advantage of the hydrostatic principle of Pascal, where the mesh repair will be reinforced by the intra-abdominal pressure [34]. Advocates of this approach have found success in the repair of different abdominal wall hernias including parastomal [35-37] and other ventral hernias [38-40].

This technique was also recently reported on the repair of lumbar hernias [32,41,42]. Sublay mesh repair is therefore advantageous as it provides tension-free repair and prevents recurrence behind the mesh, especially in primary hernias. This concurs with the post-operative findings in our patient where a quick postoperative recovery, short hospital stay, minimal analgesic demand and no recurrence at 12 months was observed. Placing the mesh in the sublay manner may be equivalent to extraperitoneal hernioplasty as advocated by enthusiasts of the minimally invasive approaches. Minimally invasive surgery has recently become a popular option for repair of lumbar hernias. One nonrandomized study demonstrated that laparoscopic lumbar hernia repairs resulted in lower operative times, hospital stay, pain medication use, as well as recovery time [43]. However, most of the laparoscopic repair was performed on patients with secondary lumbar hernias following either history of trauma or nephrectomies.

The laparoscopic approach can repair the defect at the deepest layer of the posterior abdominal wall, which allows for complete identification and coverage of the entire defect without a large incision. Nevertheless, the acquisition of expertise, the difficulty in fixing the mesh (due to the lack of strong fascia around the hernia defect), and the higher operation cost due to necessity of special instruments for mesh anchorage (e.g. tack and staple devises) may preclude laparoscopic approach becoming the prevalent option in lumbar hernia repairs. Although some authors may define laparoscopic approach as the best option [6], it is the authors' opinion that it should be reserved for centres where expertise and resources are readily available; after all, the open approach is as safe and effective.

\section{Conclusion}

This report describes a successful repair of bilateral primary lumbar hernias using open tension-free sublay prosthesis. Due to its rarity, lumbar hernias especially bilateral hernias should be treated following the principle of hernia repair by a method that has been proven safe and effective in other more commonly performed hernia repairs.

\section{References}

1. Brown J, Rogers J, Soar J (2001) Cardiac arrest during surgery and ventilation in the prone position: a case report and systematic review. Resuscitation 50(2): 233-238.

2. Burdet L, Liaudet L, Schaller MD, Broccard AF (2001) Bilateral breast necrosis after prone position ventilation. Intensive Care Med 27(8): 1435.

3. Cavallaro G, Sadighi A, Miceli M, Burza A, Carbone G, et al. (2007) Primary lumbar hernia repair: the open approach. Eur Surg Res 39(2): 88-92.

4. Moreno-Egea A, Alcaraz AC, Cuervo MC (2012) Surgical Options in Lumbar Hernia: Laparoscopic Versus Open Repair. A Long-Term Prospective Study. Surg Innov 20(4): 331-344.

5. Esposito TJ, Fedorak I (1994) Traumatic lumbar hernia: case report and literature review. J Trauma 37(1):123-126.

6. Moreno-Egea A, Baena EG, Calle MC, Martinez JA, Albasini JL (2007) Controversies in the current management of lumbar hernias. Arch Surg 142(1):82-88.

7. Barrero Candau R, Garrido Morales M (2007) [Congenital lumbar hernia and bilateral renal agenesis]. Cir Pediatr 20(2):133-135.

8. Butler BW, Shafer AD (1966) Bilateral congenital lumbar hernia. Ohio State Med J 62(6): 577-579.

9. Cocozza E, Pidoto RR, Ravera M (1999) Bilateral lumbar hernia associated with abdominal hernias. A case report. Minerva Chir 54(6): 421-433. 
10. Hickey M, Buick RG (1982) Bilateral congenital lumbar hernia. Ir J Med Sci 151(12): 388-389.

11. Zwaveling S, van der Zee DC (2012) Laparoscopic repair of an isolated congenital bilateral lumbar hernia in an infant. Eur J Pediatr Surg 22(4): 321-323.

12. Burt BM, Afifi HY, Wantz GE, Barie PS (2004) Traumatic lumbar hernia: report of cases and comprehensive review of the literature. J Trauma 57(6): 1361-1370.

13. Swartz WT (1954) Lumbar hernias. J Ky State Med Assoc 52(9): 673678.

14. Thorek M (1950) Lumbar Hernia. J Int Coll Surg 14(4): 367-393.

15. Salemis NS, Nisotakis K, Gourgiotis S, Tsohataridis E (2007) Segmental liver incarceration through a recurrent incisional lumbar hernia. Hepatobiliary Pancreat Dis Int 6(4): 442-444.

16. Faro SH, Racette CD, Lally JF, Wills JS, Mansoory A (1990) Traumatic lumbar hernia: CT diagnosis. AJR Am J Roentgenol 154(4): 757-759.

17. Losanoff JE, Kjossev KT (2002) Diagnosis and treatment of primary incarcerated lumbar hernia. Eur J Surg 168(3): 193-195.

18. Armstrong 0, Hamel A, Grignon B, JM ND, Hamel O, et al. (2008) Lumbar hernia: anatomical basis and clinical aspects. Surg Radiol Anat 30(7): 533-537.

19. Heniford BT, Iannitti DA, Gagner M (1997) Laparoscopic inferior and superior lumbar hernia repair. Arch Surg 132(10): 1141-1144.

20. Bathla L, Davies E, Fitzgibbons RJ, Cemaj S (2011) Timing of traumatic lumbar hernia repair: is delayed repair safe? Report of two cases and review of the literature. Hernia 15(2): 205-209.

21. Sommer $T$ (1999) [Lumbar intercostal hernia-a rare condition]. Ugeskr Laeger 161(48): 6640-6641.

22. Andre A, Grolleau JL, Garrido I, Guitard J, Chavoin JP (2008) [Posttraumatic lumbar hernia and abdominal wall reconstruction technique. A case report]. Ann Chir Plast Esthet 53(5): 452-456.

23. Moon HK, Dowden RV (1985) Lumbar hernia after latissimus dorsi flap. Plast Reconstr Surg 75(3): 417-419.

24. Alaedeen D (2011) Laparoscopic trans-abdominal pre-peritoneal repair of a traumatic Petit lumbar hernia with mesh. Am Surg 77(12): E292-E293.

25. Bickel A, Haj M, Eitan A (1997) Laparoscopic management of lumbar hernia. Surg Endosc 11(11): 1129-1130.

26. Burick AJ, Parascandola SA (1996) Laparoscopic repair of a traumatic lumbar hernia: a case report. J Laparoendosc Surg 6(4): 259-262.

27. Nam SY, Kee SK, Kim JO (2011) Laparoscopic transabdominal extraperitoneal mesh repair of lumbar hernia. J Korean Surg Soc 1: S74-S77.
28. Gagner M, Milone L, Gumbs A, Turner P (2010) Laparoscopic repair of left lumbar hernia after laparoscopic left nephrectomy. JSLS 14(3): 405409.

29. Ho VP, Dakin GF (2011) Laparoscopic lumbar hernia repair with bone anchor fixation. Surg Endosc 25(5): 1665.

30. Ipek T, Eyuboglu E, Aydingoz O (2005) Laparoscopic management of inferior lumbar hernia (Petit triangle hernia). Hernia 9(2): 184-187.

31. Hafner CD, Wylie JH, Brush BE (1963) Petit's lumbar hernia: repair with Marlex mesh. Arch Surg 86: 180-186.

32. Garg CP, Sharma P, Patel G, Malik P (2011) Sutureless meshplasty in lumbar hernia. Surg Innov 18(3): 285-288.

33. Solaini L, di Francesco F, Gourgiotis S (2010) A very simple technique to repair Grynfeltt-Lesshaft hernia. Hernia 14(4): 439-441.

34. Nyhus LM (1993) Iliopubic tract repair of inguinal and femoral hernia. The posterior (preperitoneal) approach. Surg Clin North Am 73(3): 487499.

35. Kasperk R, Klinge U, Schumpelick V (2000) The repair of large parastomal hernias using a midline approach and a prosthetic mesh in the sublay position. Am J Surg 179(3): 186-188.

36. Liu F, Jiye L, Yao S, Zhu Y, Yao J (2010) [In situ repair of parastomal hernia with Sublay methods in 34 cases]. Zhongguo Xiu Fu Chong Jian Wai Ke Za Zhi 24(8): 933-936.

37. Fei Y (2012) A modified sublay-keyhole technique for in situ parastomal hernia repair. Surg Today 42(9): 842-847.

38. Fei Y (2012) Compound repair of intraperitoneal onlay mesh associated with the sublay technique for giant lower ventral hernia. Ann Plast Surg 69(2): 192-196.

39. Uen YH, Wen KC (2007) An improved method for deploying the polypropylene underlay patch of the PROLENE Hernia System. Am Surg 73(5): 468-471.

40. Kurzer M, Kark A, Selouk S, Belsham P (2008) Open mesh repair of incisional hernia using a sublay technique: long-term follow-up. World J Surg 32(1): 31-36.

41. Yang F (2012) Extended Sublay repair: a modified open technique for lumbar hernias. J Plast Reconstr Aesthet Surg 65(11): 1605-1616.

42. Witherspoon J, Chakravartty S, Parry CR, Williams GL, Stephenson BM (2012) Open sutureless lumbar hernia repair using a 'memory ring' patch. Hernia 16(2): 227-228.

43. Moreno-Egea A, Torralba-Martinez JA, Morales G, Fernandez T, Girela E, et al. (2005) Open vs laparoscopic repair of secondary lumbar hernias: a prospective nonrandomized study. Surg Endosc 19(2): 184-187.

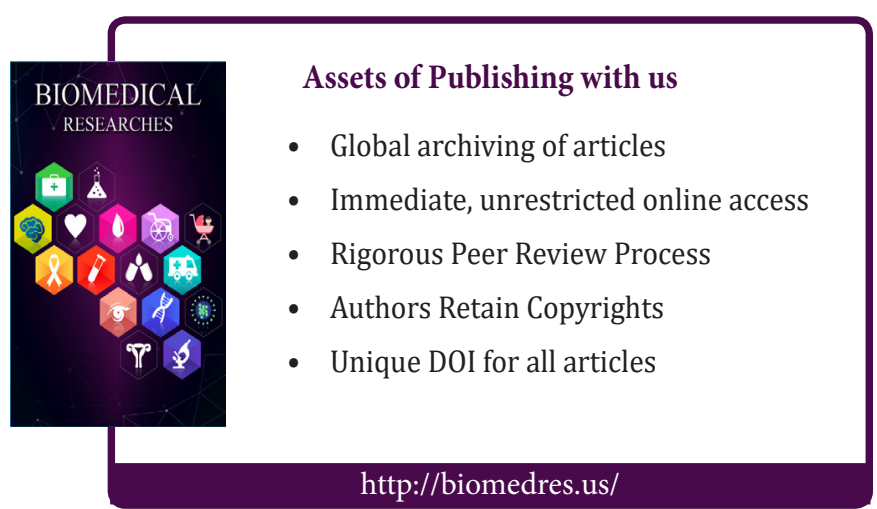

